\title{
RELACIÓN ENTRE LAS PROPIEDADES MECÁNICAS Y TRIBOLÓGICAS DE TI6AL4V ELI NITRURADO MEDIANTE DESCARGA INTENSA DE PLASMA
}

\section{RELATION BETWEEN THE MECHANICAL AND TRIBOLOGICAL PROPERTIES OF NITRIDED TI6AL4V ELI BY GLOW DISCHARGE PLASMA}

\begin{abstract}
Javier Rojas Tavera', Dario Yesid Peña Ballesteros², Hugo Armando Estupiñán Durán³
\end{abstract}
Fecha de recepción: 17 de junio de 2014

Fecha de aprobación: 8 de agosto de 2014

Referencia: J. Rojas Tavera, D.Y. Peña Ballesteros, H.A. Estupiñán Durán. (2014). Relación entre las propiedades mecánicas y tribológicas de Ti6AL4V ELI nitrurado mediante descaraga intensa de plasma. Ciencia e Ingeniería Neogranadina, 24 (2), pp. 7 - 21

\section{RESUMEN}

En este trabajo se estudió el comportamiento tribológico, la rigidez y dureza, de la superficie de la aleación Ti6AI4V ELI después de llevar a cabo un tratamiento de nitruración por descarga intensa de plasma a temperaturas de 450,480 y $520^{\circ} \mathrm{C}$. El par tribológico empleado fue metalmetal (pin de Ti6Al4V ELI - disco de Ti6Al4V ELI con tratamiento) para el respectivo cálculo del coeficiente de fricción. Los ensayos de nanoindentación se realizaron con una punta de ultra alta dureza de módulo de rigidez conocido, que permitió obtener la dureza promedio y el módulo de Young en GPa, de acuerdo con las normas ISO 14577 y ASTM G99-95 respectivamente. Para la caracterización del sustrato, se empleó microscopia electrónica de barrido, microscopia de fuerza atómica y difracción de rayos $X$, obteniendo así características importantes del comportamiento elástico-plástico, antes y después de aplicarse la nitruración a la aleación por descarga intensa de plasma.

Palabras clave: nitruración por descarga intensa de plasma, Ti6Al4V ELI, nanoindentación, tribología

\footnotetext{
1. Ing. Metalúrgico.Universidad Industrial de Santander UIS, Bucaramanga, Colombia, javiert_co@yahoo.com.

2. Ing. Metalúrgico, Ph.D, Profesor Asociado, Investigador Grupo Corrosión, Escuela de Ingeniería Metalúrgica, Universidad Industrial de Santander UIS, Bucaramanga, Colombia, dypena@uis.edu.co

3. Ing. Metalúrgico, Ph.D, Profesor Asistente, Grupo de Ciencia y Tecnología de Materiales, Departamento de materiales y minerales. Universidad Nacional de Colombia, Sede Medellín, Colombia, haestupinand@unal.edu.co
} 


\section{ABSTRACT}

This paper presents the study of the tribological behavior, rigidity and hardness, of the Ti6Al4V ELI alloy surface, after the performance of a glow discharge nitriding process at 450, 480 and $520{ }^{\circ} \mathrm{C}$. The tribological pair used was metal-metal (Ti6Al4V ELI pin - Ti6Al4V ELI pre-treated disc) for the respective calculation of the coefficient of friction. Nanoindentation tests were performed with an ultra-high hardness tip with a known stiffness modulus, which provided the average hardness and Young's modulus in GPa according to ISO 14577 and ASTM G9995 standards, respectively. Also, for the characterization of the substrate, electronic scanning microscopy, atomic force microscopy, and X-ray diffraction were used, to obtain, thus, important elasti-plastic behavioral characteristics, before and after the glow discharge plasma nitriding process was applied to the alloy.

Keywords: nitriding by glow discharge, Ti6Al4V ELI, nanoindentation, tribology

\section{INTRODUCCIÓN}

En la actualidad el desarrollo de biomateriales, en especial la aleación de Ti6Al4V ELI, está marcando el paso para la sustitucióny refuerzo de diferentes regiones del sistema óseo humano, haciendo que se conviertan en una herramienta importante para la medicina y una opción vital para pacientes que buscan mejorar su bienestar. Estos materiales contrarrestan los efectos de funcionabilidad que puedan presentar, y soportan los diferentes esfuerzos a que están sometidos, mejorando propiedades tribológicas y osteointegrándose de manera efectiva [1].

La nitruración de materiales metálicos es una técnica bien conocida para mejorar su resistencia al desgaste y a la corrosión, que disminuye el coeficiente de fricción y permite obtener mejores propiedades tribológicas [2]. Ésta se logra mediante la utilización de diferentes métodos gaseosos. Para este trabajo, se planteó la necesidad de obtener un sustrato adecuado, con una dureza aceptable, en el que no haya cambios de fase. Se sugiere la necesidad de hacer una nitruración mediante el método de descarga intensa de plasma, en la que un gas (rico en nitrógeno) recibe una descarga eléctrica luminiscente y genera iones de nitrógeno sobre el sustrato deseado, lo que favorece la difusión en el sustrato [3], en nuestro caso una aleación de Ti6Al4V ELI.

La nitruración por deposición física en fase vapor (PVD, por sus siglas en inglés) es un proceso propio de la ingeniería de materiales por el cual los iones de un material pueden ser implantados en otro sólido. Los iones implantados se distribuyen en una profundidad que oscila entre 200 y 2000 capas atómicas (0.05 $\mu \mathrm{m} 0.5 \mu \mathrm{m})$ dependiendo del tipo de ion, del material base y de la energía del bombardeo. El máximo de concentración, que para los iones ligeros como carbono o nitrógeno puede superar el $50 \%$, se sitúa a una cierta profundidad y luego disminuye suavemente [4].

El mecanismo de descarga del gas de nitruración asistida implica un complejo proceso de difusión, que es una combinación de la difusión reactiva 
a través del sustrato, en la que se da la difusión de nitrógeno en el Ti6Al4V ELI, y la posterior difusión reactiva de los nitruros formados con los componentes de la aleación.

El aumento de la nitruración alcanzada en los procesos asistidos de descarga luminiscente, que durante mucho tiempo se ha informado [5], se explica por el hecho de que aquí la superficie de nitruración se calienta más rápidamente gracias al bombardeo de iones. Se observó que, durante el proceso de revestimiento iónico, el 'bombardeo o granallado' realizado por los iones que penetran, a una profundidad nanométrica, las capas formadas afecta a la densidad, composición de la fase y la microestructura de estas capas [6].

Las ventajas de la técnica incluyen: (i) una mayor eficiencia de ionización; (ii) presiones de gas de operación más bajas; (iii) flujo de alta energía de iones y neutros; y (iv) el hecho de que el bombardeo de flujo puede proporcionar la energía de activación para la reacción química y la síntesis de compuestos. Estas ventajas se traducen en importantes efectos beneficiosos, tales como: (i) una menor contaminación y las superficies más limpias; (ii) nitruros altamente deseables y de control microestructural; (iii) tratamiento a temperaturas relativamente bajas; (iv) mejora de forma significativa la cinética de difusión; y (v) zonas de difusión que pueden ser producidas para servir como precursoras a los tratamientos de recubrimiento posteriores (tratamientos dúplex o tratamientos de recubrimiento funcionalmente graduadas-) que se pueden realizar en el mismo sistema [7].

El objetivo del presente trabajo fue el de proporcionar las pautas necesarias para analizar el comportamiento nanomecánico y nanotribológico de la nitruración mediante el método de descarga intensa de plasma y proponer recomendaciones que conlleven al mejoramiento del sustrato.

\section{MÉTODO Y MATERIALES UTILIZADOS}

El material base utilizado fue el de la aleación Ti6Al4V ELI. Su composición química se presenta en la Tabla 1. Las dimensiones de las probetas utilizadas fueron de $12 \mathrm{~mm}$ de diámetro y $2 \mathrm{~mm}$ de espesor.

A estas muestras se le hizo una preparación metalográfica de desbaste y pulido con papel de carburo de silicio iniciando en \#240 y terminando en \#1200, pasándolas a paño grueso y fino, de 0,3 um y 0,05 um con solución de alúmina. Posteriormente se realizó una limpieza ultrasónica con alcohol durante 15 minutos.

Nitruración por Deposición Física en Fase Vapor (PVD, por sus siglas en inglés): La modificación de la superficie del Ti6Al4V ELI se hizo por nitruración mediante el método

Tabla 1. Composición química de la aleación de titanio Ti6Al4V obtenida por espectrometría de fluorescencia de rayos $X$ de energía dispersa.

\begin{tabular}{|lc|c|c|c|c|c|}
\hline & $\mathrm{Al}$ & $\mathrm{V}$ & $\mathrm{Fe}$ & $\mathrm{N} 2$ & $\mathrm{TI}$ \\
\hline Composición & $(\%)$ & 6,102 & 4,119 & 0.183 & 0.05 & 88,66 \\
\hline
\end{tabular}

Fuente: Andrade, 2007 [8] 
descarga intensa de plasma. Para este proceso se utilizó un equipo NitrEos Tandem System T700x900x1300 fabricado por TRATAR S.A.S, el cual tiene un volumen útil de $700 \mathrm{~mm} \times 900$ $\mathrm{mm}$ y $700 \mathrm{~mm} \times 1300 \mathrm{~mm}$ y que posee una tecnología de calentamiento de pared caliente y un rango de operación del sistema de 350 a $600{ }^{\circ} \mathrm{C}$, con fuente de potencia de $25 \mathrm{~kW}, 800$ $V$ hasta $10 \mathrm{kHz}$, y potencia pulso $70 \mathrm{~kW}$ (Figura 1). Las probetas trabajaron a una temperatura de 450,480 y $520{ }^{\circ} \mathrm{C}$, con un tiempo de permanencia de 4 horas.

Difracción de Rayos X (DRX): La caracterización mediante difracción de rayos $X(D R X)$ se realizó en un difractómetro marca BRUKER modelo D8 DISCOVER con Geometría DaVinci. El análisis se realizó con haz rasante. La muestra fue irradiada con un haz monocromático y a partir de las intensidades a muy bajo ángulo se obtiene información sobre tamaño, distribución de tamaño de partículas, forma de partículas y estructura interna. Además, se permite evaluar tamaños comprendidos entre 0,5 y $50 \mathrm{~nm}$ en diferentes clases de materiales.

Nanoindentación: La prueba de indentación consiste en presionar un indentador sobre la superficie dejando una marca o huella sobre el material. El instrumento de alta resolución registra continuamente la carga, $\mathrm{P}$, y la profundidad de penetración, h, usando un penetrador que en la presente investigación fue un nanoindentador de punta Berkovich. Estos datos se utilizaron para obtener la carga frente a la profundidad de penetración de la curva ( $P$ vs. h). Las cantidades físicas

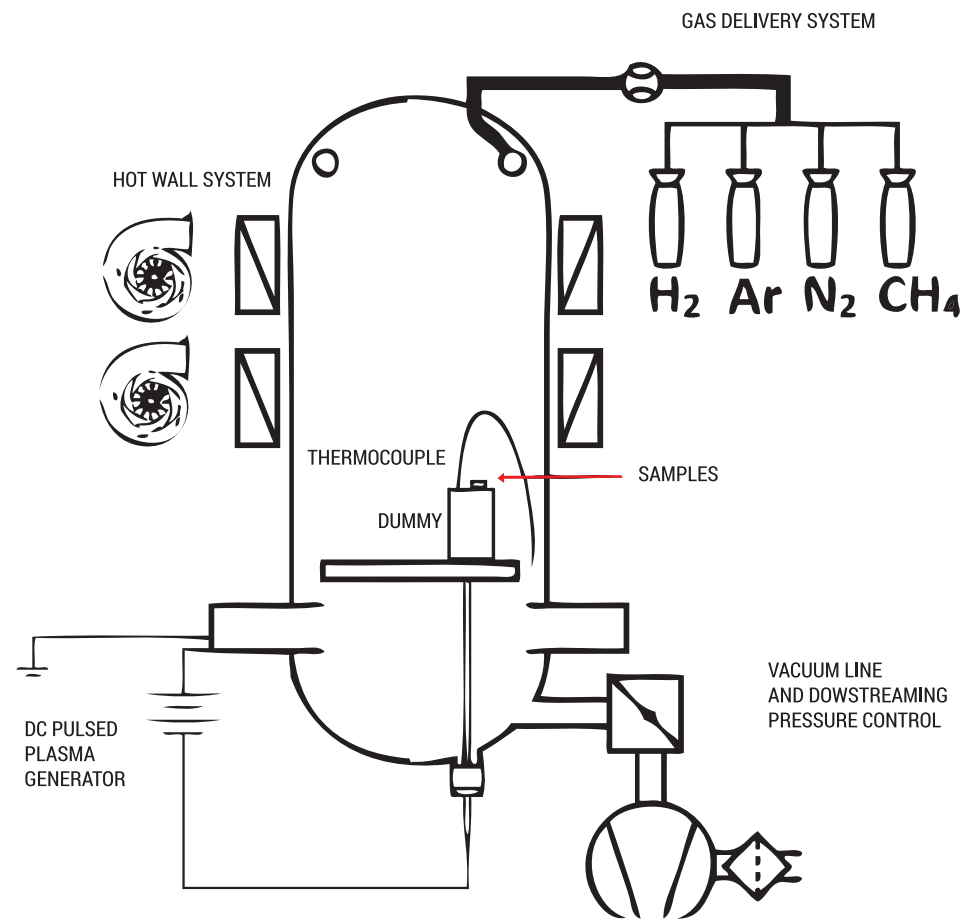

Figura 1. Esquema general del reactor utilizado para la nitruración. 
importantes obtenidas a partir de la carga frente a la profundidad de penetración son: la carga máxima, Pmax, la profundidad de penetración máxima, Hmax, la profundidad de penetración final y la rigidez de contacto. De acuerdo con el modelo O-P que es el método más comúnmente utilizado para obtener la dureza y el módulo Young de un material por nanoindentación instrumentada, la nanodureza, $\mathrm{H}$, está expresada como:

$$
H=P \max / A c r
$$

Donde Pmax es la carga máxima aplicada y $A c r$ es el área verdadera de contacto entre el penetrador y el material.

El equipo Nanotec cuenta con un nanoindentador XP de MTS. En este nanoindentador la carga se aplica mediante una bobina electromagnética calibrada con una resolución de $50 \mathrm{nN}$. El desplazamiento que experimenta el indentador se mide mediante un transductor capacitivo con una resolución de $0,01 \mathrm{~nm}$.

El método de cálculo que se empleó para determinar estas propiedades se basó en los estudios realizados por Oliver y Pharr [9], que a su vez se basaron en los trabajos desarrollados por Hertz, Sneddon, y Doerner y Nix [1011] acerca del contacto elástico entre un indentador de geometría conocida y un plano elástico. La carga aplicada para este trabajo fue de $2 \mathrm{mN}$ y las indentaciones se hicieron con una separación de 10 micras para cada muestra.

Tribología: El equipo utilizado para la prueba de pin sobre disco fue construido de acuerdo a la norma ASTM G99-95 y que actualmente posee la Universidad Nacional de Medellín. El equipo utiliza el método estándar ASTM G 99 - 95A para pruebas de desgaste. La balanza que se utilizó para pesar las probetas fue una Sartoruis CP225 D, que tiene una resolución de $+/-0,01 \mathrm{mg}(0,00001 \mathrm{~g})$ para masas menores a $80 \mathrm{~g}$, y para masas de $80-220 \mathrm{~g}$ ( $220 \mathrm{~g}$ es el máximo) la resolución es de 0,1 mg (0001 g), y el baño ultrasónico es un Elma LC $30 \mathrm{H}$.

Los parámetros bajo los cuales se realizaron las pruebas fueron: una carga de $0,5 \mathrm{Kg}-\mathrm{F}$, una velocidad de $0,05 \mathrm{~m} / \mathrm{s}$, durante un tiempo de 30 min y con 77 revoluciones por minuto (RPM). Inicialmente se pesaron los pines de Ti6Al4VELI y las probetas de estudio. Una vez realizada la prueba, se procedió a pesar los pines y las probetas de Ti6Al4V-ELI nuevamente y se evaluaron respecto a las condiciones iniciales. Finalmente se analizaron estos datos respecto a los parámetros establecidos.

\section{RESULTADOS Y DISCUSIÓN}

\section{Caracterización de blanco y sustrato} por nitruración: Se realizó la prueba de nanoindentación, utilizando un equipo marca Nanotec, en la que se evidenció el aumento deseado de la dureza con respecto a la aleación sin tratamiento, después de haber realizado la nitruración mediante descarga intensa de plasma. En este punto surge la necesidad de plantear la posible forma o modelo de implantación de nitrógeno en la aleación, incrementando la dureza del sustrato.

Esto se debe a que al tener alta temperatura (por el método descarga intensa de plasma), la energía de ionización aumenta, aumentando así la posibilidad de una mayor descarga ionizante (bombardeo iónico) sobre la superficie y por consiguiente mayor difusión de nitrógeno. 
Al obtener una elevada densidad de productos sobre la superficie de la aleación lo que se interpreta es que el factor de empaquetamiento aumenta, disminuyendo la distancia interatómica y, por consiguiente, disminuyendo la energía potencial, que es la responsable de tener unidos los átomos. La consecuencia de lo anterior es el anclaje de dislocaciones producido por la difusión del nitrógeno en el sustrato del Ti6Al4V ELI, lo cual confirma el aumento de la dureza del material [12] e indica que el nitrógeno llegó a modificar la red de la aleación sin deformar notoriamente su microestructura, pero brindando el cambio deseado y esperado [12-13].

El nitrógeno incorporado en la red o microestructura de la aleación no se detectó por SEM realizado después del decapado, pero sí antes de éste (Figura 2), lo cual puede indicar que el nitrógeno incorporado a la red es elemental y de difícil detección por esta técnica.

Bajo el efecto del campo eléctrico se mejora la actividad de los átomos de nitrógeno al ser afectada por los iones de alta energía y se incrementa la eficiencia de nitruración. Además, los iones con cargas positivas tienen una tendencia a moverse hacia el cátodo. Por lo tanto, la pulverización catódica de las muestras de la aleación de Ti6Al4V ELI se inhibe eficazmente, la integridad de la superficie se mejora y la rugosidad de la superficie se reduce obviamente [14]. Esta técnica es razonable para obtener una capa de nitruración con alta resistencia al desgaste y baja rugosidad de la superficie mediante el uso del fenómeno de descarga de cátodo asistido, cuando existen átomos de nitrógeno activos intensivos y bombardeos de iones débiles simultáneamente [14].

En el análisis realizado por DRX, los posibles cambios generados por el tratamiento de nitruración en la microestructura podrían manifestarse a escalas muy pequeñas en la superficie y, por lo tanto, era necesario considerar una óptima evaluación para encontrar fases cristalinas en formación.

La Figura 3 demuestra lo anteriormente expuesto y confirma lo inicialmente descrito

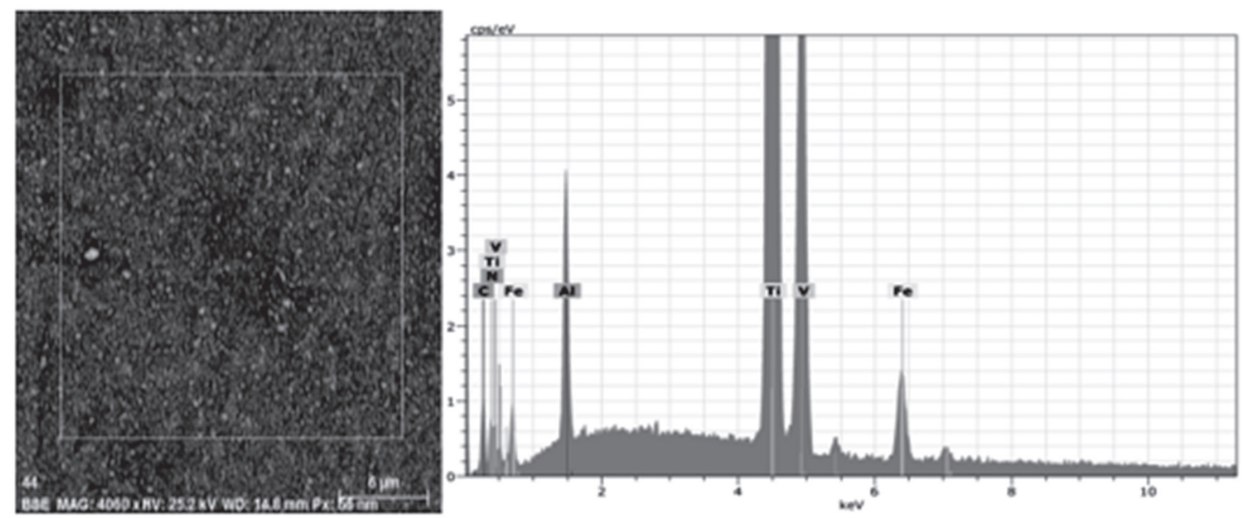

Figura 2. MEB realizado a la muestra inmediatamente después de la deposición de nitrógeno (U.N. de Medellín). 


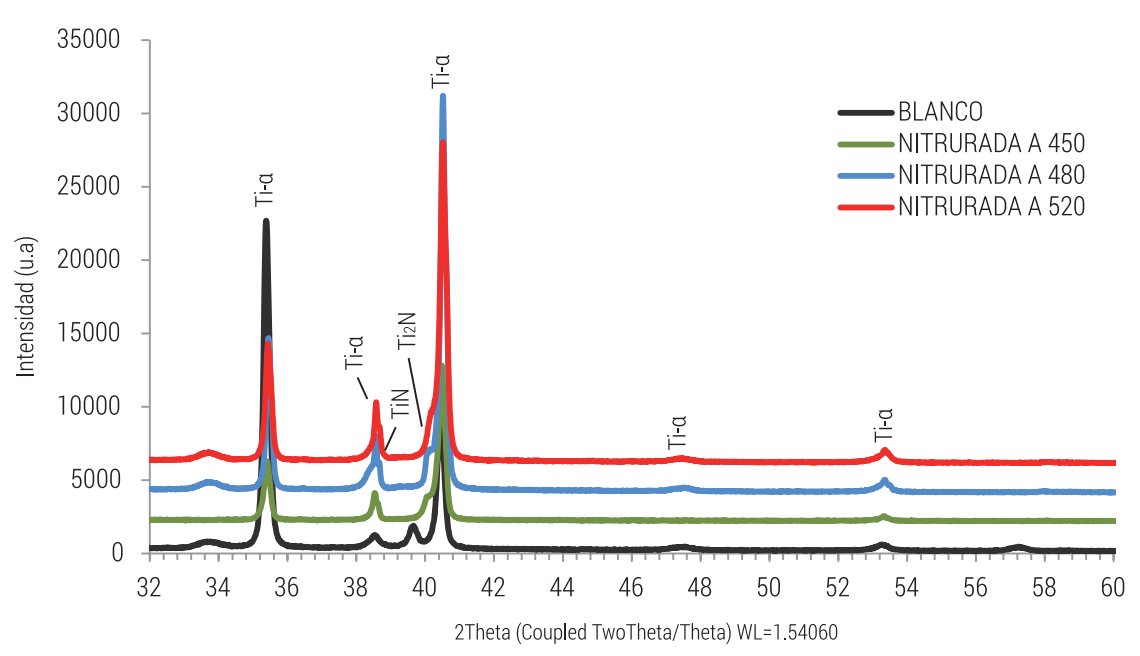

Figura 3. Superposición de los perfiles de difracción haz rasante.

en la difusión del nitrógeno. Bajo el efecto del campo eléctrico se mejora la actividad de los átomos denitrógenoal serafectadaporlosiones de alta energía y se incrementa la eficiencia de nitruración. Esto permite identificar pequeñas modificaciones o desplazamientos, producto de los cambios en los parámetros de red inducidos por el nitrógeno, que fue incorporado en la microestructura [15-16].

La aparición de nitruros a bajas temperaturas (para nuestro caso entre 450 y $520^{\circ} \mathrm{C}$ ) inciden directamente en la dureza obtenida por el sustrato y es confirmada por otros autores que obtuvieron nitruros por debajo de los $773 \mathrm{~K}, \epsilon-\mathrm{Ti} 2 \mathrm{~N}$ y $\delta$-TiN los cuales están claramente presentes para una atmósfera $\mathrm{N}$ / $\mathrm{H}=3 / 2$, después de un tratamiento de más de $240 \mathrm{~min}$ [17].

El efecto de la descarga intensa de plasma en la composición sobre la superficie del Ti$6 \mathrm{Al}-4 \mathrm{~V} \mathrm{ELI}$ es una función muy compleja de los parámetros del proceso: tiempo de tratamiento, la proporción de nitrógeno, temperatura de la muestra y la presión total durante el tratamiento, corroborando variables descritas por otros autores [17].

Así mismo en el MFA se realizó la topografía en un equipo diCP II Veeco en modo contacto, con un área de trabajo $50 \mu \mathrm{m} \times 50 \mu \mathrm{m}$. En la Figura 4 se muestra la topografía de las muestras, en la cuales se evidencia un notable cambio de rugosidad.

Comparando entre las muestras sin tratamiento de nitruración con las nitruradas, se observa que los sustratos nitrurados disminuyen su rugosidad con el incremento de la temperatura. Este es el efecto que produce la incorporación de nitrógeno en el sustrato. Para los cálculos respectivos de la Tabla 2 se realizaron varios perfiles de rugosidad en distintas direcciones con la medición de microscopia de fuerza atómica.

Estos perfiles sirvieron como base para establecer la desviación estándar y la desviación media aritmética de RMS y Ra, los cuales son calculados por medio de las ecuaciones (2): 
(a)

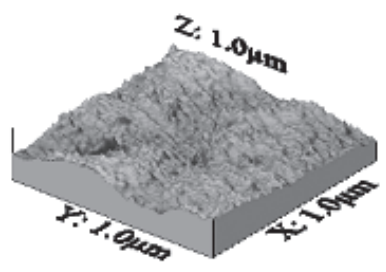

(c)

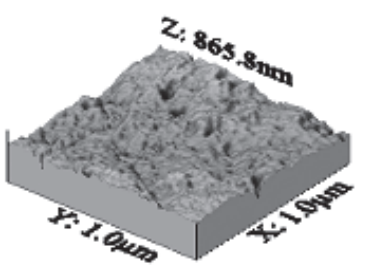

(b)

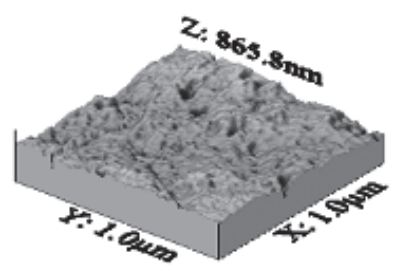

(d)

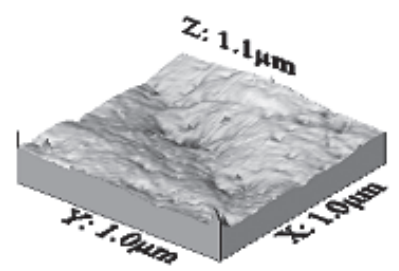

Figura 4. Micrografías MFA de probetas nitruradas: a) blanco b) $450^{\circ} \mathrm{C}$, c) 480 y d) $520^{\circ} \mathrm{C}$

Tabla 2. Valores de rugosidad RMS y Ra para las diferentes muestras.

\begin{tabular}{|c|c|c|}
\hline Muestras & RMS (nm) & Ra $(\mathrm{nm})$ \\
\hline Blanco & 70,25 & 69,47 \\
\hline Nitrurada a $450^{\circ} \mathrm{C}$ & 63,91 & 49,22 \\
\hline Nitrurada a $480^{\circ} \mathrm{C}$ & 39,30 & 31,57 \\
\hline Nitrurada a $520^{\circ} \mathrm{C}$ & 29,29 & 23,19 \\
\hline
\end{tabular}

$$
R a=\frac{1}{l m} \int_{0}^{l m}|y| d x \quad R q=\sqrt{\frac{1}{l m} \int_{0}^{l m} y^{2}(x) d x}
$$

La Figura 5 muestra las gráficas de seguida de una descarga. Estas curvas no son nanoindentación con la variación de la lineales. Los datos de descargas se utilizan para temperatura. Ésta indica la forma en que se determinar las propiedades mecánicas sobre realizólapruebadecargahastaunpuntomáximo, la base de la teoría de indentación, en la cual 


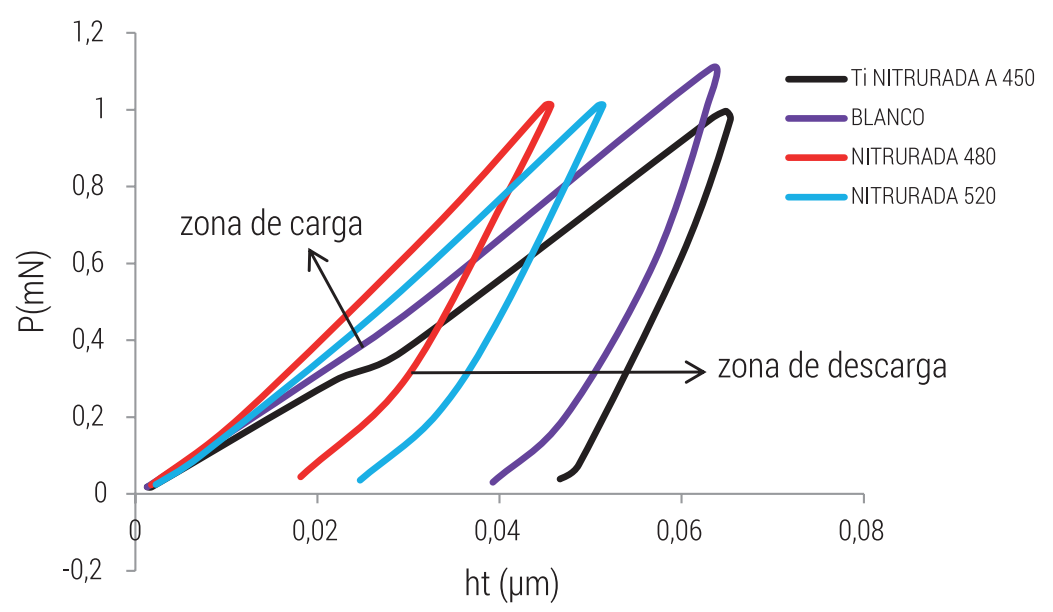

Figura 5. Graficas de nanoindentación P vs. ht.

la porción de la descarga inicial de la curva de carga-profundidad representa la recuperación puramente elástica [19].

La teoría desarrollada para el cálculo de dureza mediante ensayos de nanoindentación supone que el material es idealmente elastoplástico y que el material se hunde alrededor del indentador [18]. A partir de la curva de cargaprofundidad, el módulo elástico y la dureza se calculan sobre la base de los métodos propuestos por Oliver y Pharr [19]. Las ecuaciones utilizadas son:

$$
E_{r}=\frac{\sqrt{\pi} S}{2 \sqrt{A}}
$$

Donde A es el área de contacto del penetrador, y Er es el módulo reducido. Éste se define por:

$$
\frac{1}{E_{r}}=\frac{\left(1-v_{m}^{2}\right)}{E_{m}}+\frac{\left(1-v_{i}^{2}\right)}{E_{i}}
$$

Donde Em y Vm son el módulo de Young y el coeficiente de Poisson del material indentado respectivamente, y Ei y Vi los del indentador. La dureza de la aleación $\mathrm{H}$, se define como la carga máxima, Pmax, dividido por el área proyectada de la indentación bajo esta carga, es decir, $H=$ Pmax / Amax. (1)

Al comparar el estado inicial del sustrato sin nitrurar con el sustrato nitrurado se observa un aumento de la dureza, lo que cumple en principio con el propósito de la investigación de obtener una dureza adecuada, entre 6,4 $\pm 0,4$ y 9,8 \pm $0,4 \mathrm{GPa}$, ver Figura 6. Una dureza más elevada, por encima de $10 \mathrm{GPa}$, hace que la superficie sea muy rígida, no amortiguando y haciendo que la carga no se distribuya adecuadamente, con lo cual no lograría un mejor acople entre el material base y el recubrimiento [20], (un sustrato muy rígido ocasionaría fragilidad en la capa nitrurada).

Así mismo, se observa una disminución en el módulo de Young, Tabla 3, lo cual indica que aumenta su comportamiento plástico y su capacidad de resistir cargas sin deformarse, 


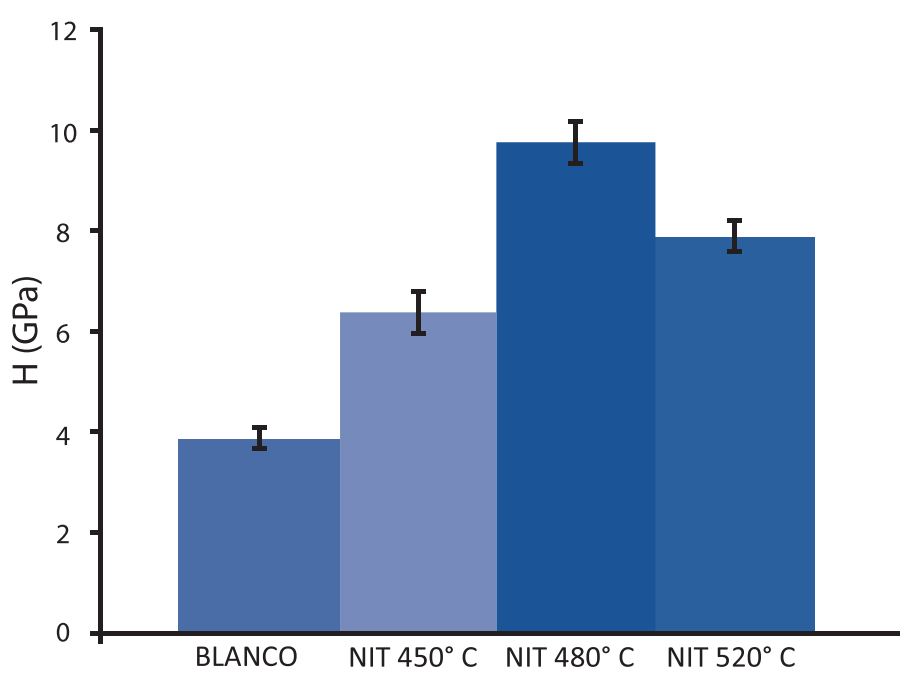

Figura 6. Dureza obtenida por nanoindentación en GPa.

Tabla 3. Dureza y Módulo de Young obtenidos por nanoindentación.

\begin{tabular}{|c|c|c|c|}
$\begin{array}{c}\text { No. de } \\
\text { Probeta }\end{array}$ & Tipo de Tratamiento & $\begin{array}{c}\text { Dureza por } \\
\text { Nanoindentación } \\
\text { GPa }\end{array}$ & $\begin{array}{c}\text { Módulo de Young } \\
\text { GPa }\end{array}$ \\
\hline Ti-1 & SIN NITRURAR & $3,9 \pm 0,2$ & $271,8 \pm 25,4$ \\
\hline Ti-2 & $\begin{array}{c}\text { NITRURADA } \\
450^{\circ} \mathrm{C}\end{array}$ & $6,4 \pm 0,4$ & $197,7 \pm 8,1$ \\
\hline Ti-3 & $\begin{array}{c}\text { NITRURADA } \\
480^{\circ} \mathrm{C}\end{array}$ & $9,8 \pm 0,4$ & $161,7 \pm 6,1$ \\
\hline Ti-4 & $\begin{array}{c}\text { NITRURADA } \\
520^{\circ} \mathrm{C}\end{array}$ & $7,9 \pm 0,3$ & $145,5 \pm 2,4$ \\
\hline
\end{tabular}

aumentando su capacidad de recuperación [21]. Esto, debido a que disminuye la energía potencial, suponiendo una disminución en las distancias interatómicas del material base Ti6Al4V ELI, lo que logra disminuir su rigidez y evita que cuando sea sometida a grandes esfuerzos, soporte la delaminación y la producción de partículas en forma de debris, desacelerando así su degradación.

Los resultados de la prueba de desgaste de las muestras después de ser sometidas a la prueba de pin sobre disco se relacionan en la Tabla 4, e indican que la pérdida de masa disminuye a medida que aumenta la temperatura de nitruración por descarga intensa de plasma, debido al mecanismo de nitruración, en el cual hay un bombardeo iónico de los iones activos ( $\mathrm{Ar}$ $\rightarrow \mathrm{Ar}++\mathrm{e}, \mathrm{N} 2 \rightarrow \mathrm{N}++\mathrm{e}, \mathrm{N} 2 \rightarrow \mathrm{N}$ ), introduciendo deficiencias o vacancias en la superficie de la muestra por la difusión del nitrógeno, bajo el efecto térmico a que se encuentra sometida la muestra [14]. Esto conlleva a un aumento del factor de empaquetamiento, disminuyendo 
Tabla 4. Coeficiente de fricción y pesos después de efectuada la prueba de pin sobre disco.

\begin{tabular}{|c|c|c|c|c|c|}
\hline $\begin{array}{c}\text { No. De } \\
\text { Probeta }\end{array}$ & $\begin{array}{c}\text { Tipo de } \\
\text { Tratamiento }\end{array}$ & $\begin{array}{c}\text { Peso de } \\
\text { Probeta Inicial } \\
\text { gr }\end{array}$ & $\begin{array}{c}\text { Peso de } \\
\text { Probeta } \\
\text { Final } \\
\text { gr }\end{array}$ & $\begin{array}{c}\text { Diferencia } \\
\text { gr }\end{array}$ & $\begin{array}{c}\text { Coeficiente de } \\
\text { Fricción } \\
\boldsymbol{\mu}\end{array}$ \\
\hline Ti-1 & $\begin{array}{c}\text { SIN } \\
\text { NITRURAR }\end{array}$ & 1.35334 & 1.35255 & $7,9 \mathrm{E}-4 \pm 1 \mathrm{E}-5$ & $0,78 \pm 0,02$ \\
\hline Ti-2 & $\begin{array}{c}\text { NITRURADA } \\
450^{\circ} \text { C }\end{array}$ & 1.29155 & 1.29072 & $8,3 \mathrm{E}-4 \pm 1 \mathrm{E}-5$ & $0,29 \pm 0,02$ \\
\hline Ti-3 & $\begin{array}{c}\text { NITRURADA } \\
480^{\circ} \text { C }\end{array}$ & 1.29749 & 1.29675 & $7,4 \mathrm{E}-4 \pm 1 \mathrm{E}-5$ & $0,34 \pm 0,01$ \\
\hline Ti-4 & $\begin{array}{c}\text { NITRURADA } \\
520^{\circ} \text { C }\end{array}$ & 1.31151 & 1.31092 & $5,9 \mathrm{E}-4 \pm 1 \mathrm{E}-5$ & $0,32 \pm 0,02$ \\
\hline
\end{tabular}

la distancia interatómica, y por consiguiente, disminuyendo la energía potencial, la cual es la responsable de tener unidos los átomos. La consecuencia de lo anterior es el anclaje de dislocaciones producida por la difusión del nitrógeno en el sustrato del Ti6Al4V ELI, lo cual confirma el aumento de la dureza del material, la disminución del coeficiente de fricción y consecuentemente la disminución en la pérdida de masa [12] (ver Figura 7). Las imágenes de SEM de las pistas de desgaste se presentan en la Figura 9.
El tratamiento denitruración por descarga intensa de plasma conduce a un buen comportamiento tribológico, reduciendo el desgaste y la fricción de la aleación Ti6Al4V ELI, lo cual se traduce en baja pérdida de masa. Este comportamiento también lo reportan otros autores, incluso bajo altas cargas aplicadas (hasta 35N) [22].

El coeficiente de fricción de la muestra no tratada varió de 0,6 a 1,2 inicialmente, estabilizándose al final de la prueba entre 0,78 $\pm 0,02$ (Figura 8). Este coeficiente es elevado en comparación con

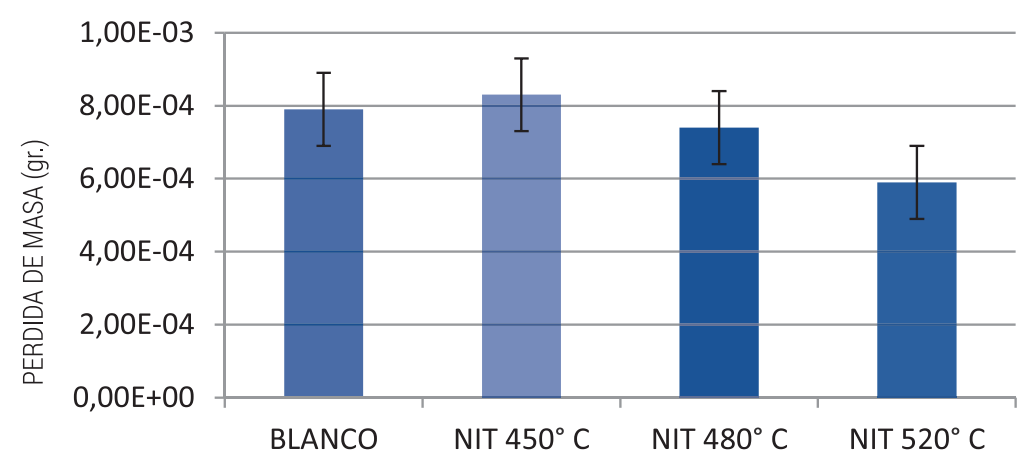

Figura 7. Pérdida de masa (en gr) obtenida en prueba de pin sobre disco. 


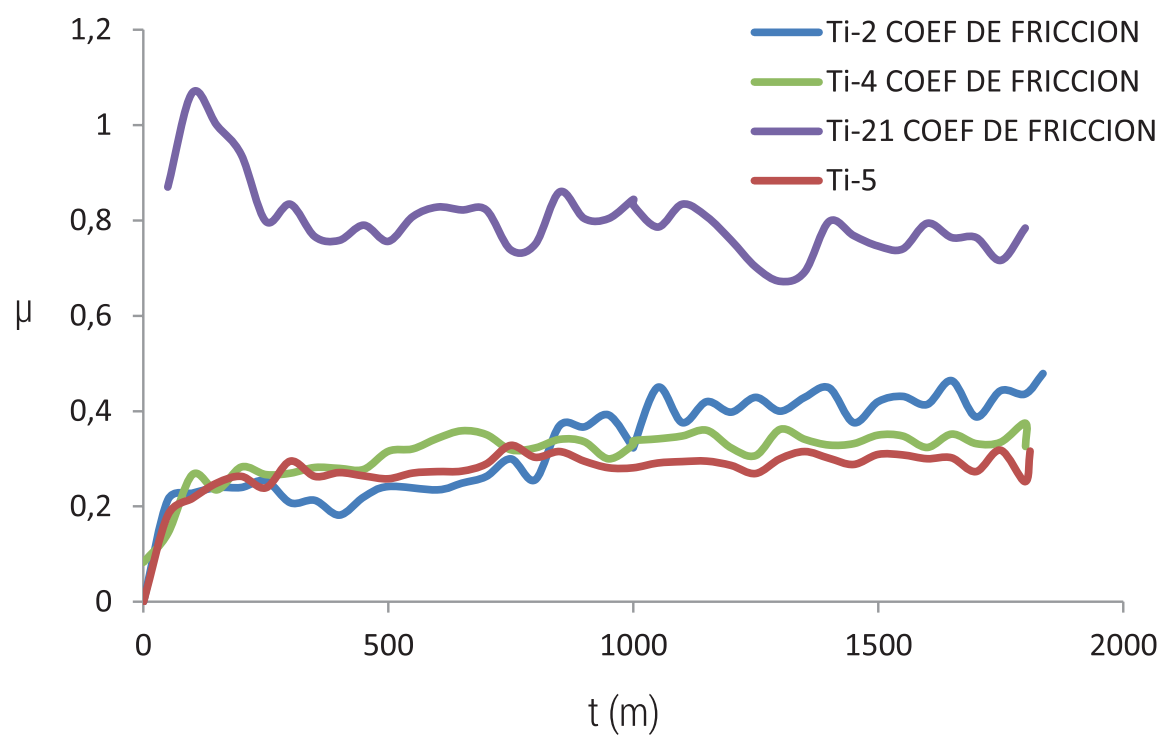

Figura 8. Comportamiento de la fricción con respecto al tiempo.
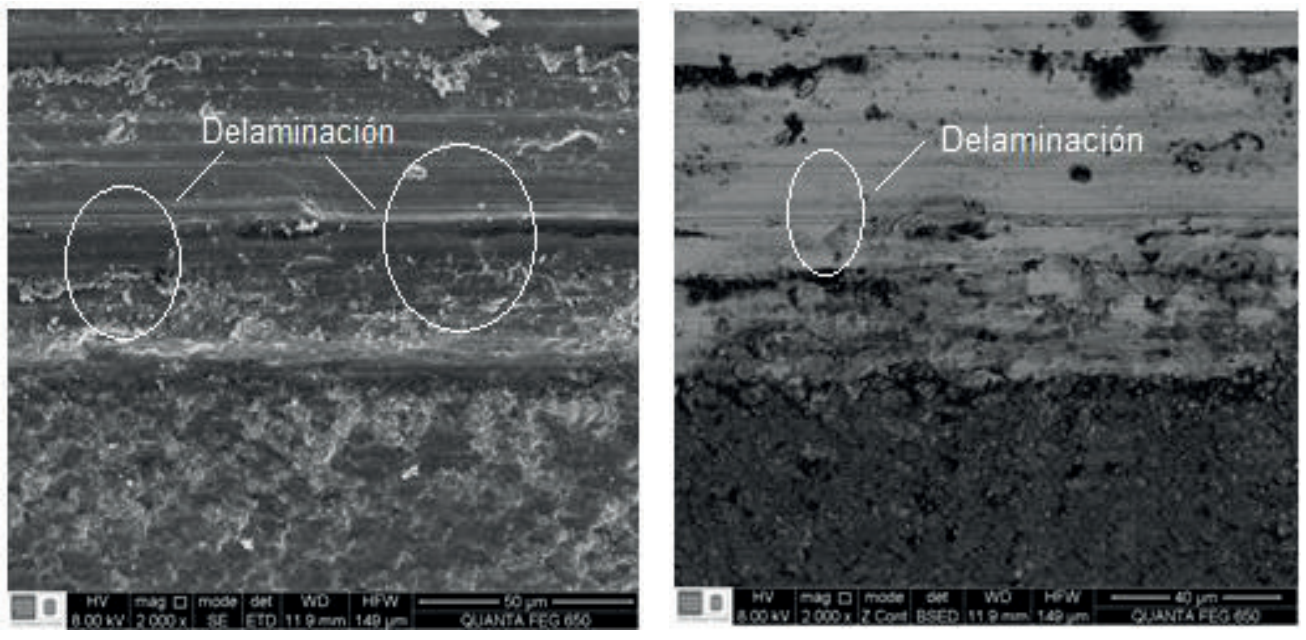

Figura 9. SEM obtenido de las muestras nitruradas por PVD y la muestra no nitrurada.

las muestras nitruradas, que presentaron un bajo coeficiente de fricción, lo cual indica que el tratamiento utilizado es adecuado y disminuye a medida que aumenta la temperatura [23].
Estos valores indican la oposición que en principio opuso el material nitrurado por descarga intensa de plasma, pero que al final terminó cediendo por la forma en que se 
trataban de separar las partículas (forma de láminas, Figura 9), lo que explica así la poca cantidad de masa que perdió el sustrato. Es interesante observar que el recubrimiento por descarga intensa de plasma mostró una zona de transición de deslizamiento, de un régimen de baja fricción y desgaste insignificante a un régimen de fricción y desgaste moderado. Esto indica que la forma en que se produce este desgate podría estar relacionada con la vida de los revestimientos en sí [22].

Para la muestra sin nitrurar, la masa perdida calculada es de aproximadamente 0,00079 \pm $0,01 \mathrm{mg}(0,00001 \mathrm{~g})$, ver Tabla 3. El coeficiente de fricción de las muestras nitruradas (Figura 8) varía de aproximadamente 0,155 a aproximadamente 0,337 inicialmente, estabilizándose al final en 0,276 a 0,290 (para las muestras nitruradas a 450 y $520^{\circ} \mathrm{C}$ ) y de 0,155 a 0,337 inicialmente, estabilizándose al final de la prueba en 0,419 a 0,396 (para la muestra nitrurada a $480^{\circ} \mathrm{C}$ ). El peso perdido calculado es de aproximadamente 0.00083 $\mathrm{g} \pm 0,01 \mathrm{mg}(0,00001 \mathrm{~g})$ para la muestra nitrurada a $450{ }^{\circ} \mathrm{C}$, de $0.00074 \mathrm{~g} \pm 0,01 \mathrm{mg}(0,00001 \mathrm{~g})$ y de $0.00059 \mathrm{~g} \pm 0,01 \mathrm{mg}(0,00001 \mathrm{~g})$. Por lo tanto, las pruebas indican que el comportamiento de la resistencia al desgaste de las muestras nitruradas es mejor que el de la muestra no tratada, lo que indica a su vez que la temperatura favorece las propiedades tribológicas de la aleación Ti6Al4V F136, siendo de éstas la mejor de $520^{\circ} \mathrm{C}$.

\section{CONCLUSIONES}

El proceso de nitruración mediante descarga intensa de plasma favorece la oxidación de la aleación Ti6Al4V ELI, lo que conlleva a un alisamiento o disminución de la rugosidad, tal como se verificó en los resultados obtenidos por microscopia de fuerza atómica MFA. Esto indica que es posible que se favorezca la ubicación del oxígeno en los puntos de más baja energía, lo cual mejora sus propiedades tribológicas.

La variación del módulo de Young y el incremento de la dureza superficial de la aleación Ti6Al4V ELI conlleva al aumento de la zona plástico-elástica, lo que garantiza que el nivel o capacidad de deformación aumente y mejora así su capacidad de resistir esfuerzos de tensión y compresión. Esto confirma que el método utilizado de nitruración por deposición física en fase vapor mediante descarga intensa de plasma dio como resultado los efectos esperados, mejorando así sus propiedades mecánicas, y por consiguiente su utilización y estudio en futuros remplazos para prótesis del cuerpo humano.

El tratamiento de nitruración por descarga intensa de plasma conduce a un buen comportamiento tribológico al reducir el desgaste y la fricción de la aleación Ti6Al4V ELI, lo cual se traduce en baja pérdida de masa a medida que se incrementa la temperatura. Este comportamiento está estrechamente ligado con la disminución del coeficiente de fricción, el aumento de la zona elástico-plástica y la pérdida de masa en forma de láminas (mecanismo de delaminación), evitando así el debris.

\section{REFERENCIAS BIBLIOGRÁFICAS}

[1] Cunningham, B.W., Hu, N., Zorn, C.M. \& McAfee, P.C. (2009). Bioactive titanium calcium phosphate coating for disc arthroplasty: analysis of 58 vertebral end plates after 6- to 12-month implantation. The Spine Journal, 9, pp.836-845.

[2] Fraczek, T., Olejnik, M., Jasiñski, J. \& Skuza, Z. (2011). Short-term low temperature glow discharge nitriding of 316 austenitic steel. METABK, 50(3), pp.151-154. 
[3] Kashaev, N., Stock, H.-R. \& Mayr, P. (2004). Nitriding of $\mathrm{ti}-6 \% \mathrm{al}-4 \% \mathrm{v}$ alloy in the plasma of an intensified glow discharge. Metal Science and Heat Treatment, 46(78), pp.294-298.

[4] Sun, Y. \& Bell, T. (1997). A numerical model of plasma nitriding of low alloy steel. Materials Science and Engineering, 224(1-2), pp.33-47.

[5] Lachtin, J., Krymskij, J. \& Sernionov, P.A. (1964). Metalloviedienie i termiceskaja obrabotka metallov. Moscú, Rusia.

[6] Robertson, J. (1993). Deposition mechanisms for promoting sp3 bonding in diamond-like carbon. Diamond and Related Materials, 2(5-7), pp.984-989.

[7] Meletis, E.I. (2002) Intensified plasmaassisted processing: science and engineering. Surface and Coating Technology, 149, pp.95-113.

[8] Andrade, M.J. (2007). Comportamiento electroquímico de aleación de Ti6Al4V anodizadaensoluciónde $\mathrm{NaH} 2 \mathrm{PO} 4.2 \mathrm{H} 2 \mathrm{O}$. Scientia et Technica Año XIII, 36, pp.215220.

[9] Oliver, W.C., Pharr, G.M. (1992). "An improved technique for determining hardness and elastic modulus using load and displacement sensing indentation experiments," J. Mater. Res., 7, pp.15641583.

[10] Hertz, H. (1982). On the contact of rigid elastic solids and on hardness. Verhandlungen des Vereins zur Beförderung des Gewerbefleisses. Leipzig, pp.90-156.
[11] Sneddon, I.N. (1882). The relation between load and penetration in axisymmetric business problem for a punch of arbitrary profile. International Journal of Engineering Science, 3, pp.47-57.

[12] Askeland, D.R. (1998). Ciencia e ingeniería de los Materiales. $3^{a}$ Edición. International Thomsom Editores, pp.92-97,107-109.

[13] ASM Comitte of Metallography of titanium and titanium alloys. (1980). Microestructure of titanium and titanium alloys. Handbook eighthedition, American Society of Metals, no 7, pp.312-344.

[14] Tang, J.G., Liu, D.X., Tang, C.B. \& Zhang, X.H. (2013). Surface modification of Ti-6Al-4V alloy by cathode assisting discharge setup and conventional plasma nitriding methods. Science China, 56(8), pp.1858-1864.

[15] Khalaf, M.K., Chiad, B.T., Al-Zubaydi T.L. \& Alani, Z.T. (2011). Surface Modification of Ti6Al4V Alloy by Glow Dicharge-Plasma Nitriding. Iraqi Journal of Physics, 9(16), pp. 34-40.

[16] Brading, H.J. (1992). Plasma nitriding with Nitrogen, Hydrogen, and Argon Gas Mixture. Surface Engineering, 8(3), p.207.

[17] da Silva, S.L.R., Kerber, L.O., Amaral, L. \& dos Santos, C.A. (1999). X-ray diffraction measurements of plasma-nitrided $\mathrm{Ti}-$ 6Al-4V. Surface and Coatings Technology, 116-119, pp.342-346.

[18] Meza, J.M., Chaves, C.A. \& Vélez, J.M. (2006). Técnicas de indentación: medición de propiedades mecánicas en cerámicas. DYNA, Journal of the Facultad de Minas, 
Universidad Nacional de Colombia - [21] Bemporad, E., Sebastiani, M., Staia, M.H. Medellín Campus, 73(149). \& Puchi Cabrera, E. (2008). Tribological studies on PVD/HVOF duplex coatings

[19] Tang, C.Y., Tsui, C.P., Janackovic, D.J. \& Uskokovic, P.S. (2006). Nanomechanical properties evaluation of bioactive glass coatings on titanium alloy substrate. Journal of Optoelectronics and Advanced Materials, 8(3), pp.1194 $-1199$

[20] King, E., Fernández González, B.J., de Damborenea González, J.J. \& Ruiz Fernández, J. (2000). Nitruración gaseosa a elevada temperatura de la aleación Tí6Al4V para mejorar su resistencia al desgaste. Revista de Metalurgia, 36, pp.403-409. on Ti6Al4V substrate. Surface \& Coatings Technology, 203, pp.566-571.

[22] Ceschini, L., Lanzoni, E., Martini, C., Prandstraller, D. \& Sambogna, G. (2008). Comparison of dry sliding friction and wear of Ti6Al4V alloy treated by plasma electrolytic oxidation and PVD coating. WEAR, 264(1-2), pp.86-95.

[23] Barbieri, F.C., Otani, C., Lepienski, C.M., Urruchi, W.I., Maciel, H.S. \& Petraconi, G. (2002). Nanoindentation study of Ti6Al4V alloy nitrided by low intensity plasma jet process. Vacum, 67, pp.457-461. 